\title{
Arborização em canteiros centrais na cidade de Patos, Paraíba
}

\section{Arborization in the medians of the city of Patos, Paraíba Estate}

\author{
Juliane Neves de Lucena ${ }^{1}$, Patrícia Carneiro Souto ${ }^{2}$; Jorge Danilo Zea Camaño ${ }^{3}, J_{a c o b}$ Silva Souto ${ }^{4}$ Lauter Silva Souto ${ }^{5}$
}

Resumo: A arborização de canteiros nas cidades é uma prática que embeleza e permiti reduzir efeitos antropogênicos. O trabalho objetivou quantificar e avaliar a qualidade das árvores localizadas em canteiros centrais da cidade de Patos-PB e o risco que elas representam para a população. Foi realizado o levantamento florístico em 1.391,95 m de canteiros centrais, registrando informações dendrométricas e qualitativas quanto aos aspectos das condições existentes no meio. Foram registrados 166 indivíduos representados em 14 espécies, sendo 71,4\% exóticas com destaque para Azadirachta indica e Prosopis juliflora. A maioria das árvores são adultas e medem mais de $10 \mathrm{~cm}$ de DAP com altura variando entre 1,6-7,21 m. Em relação à avaliação do risco, não registrou-se indivíduos em conflito com as redes elétricas e sim a presença de galhos ocos nos níveis médio e leve. Na maioria dos indivíduos o sistema radicular apresenta-se subterrâneo, mas, observou-se que 70,1\% não apresentam neilóides em superfície, aumentando a suscetibilidade de risco de queda desses indivíduos. Conclui-se que as espécies de maior ocorrência nos canteiros centrais da cidade de Patos são exóticas e que a maioria dos indivíduos sofreram intervenção silvicultural de forma inadequada.

Palavras chaves: arborização viária, arboricultura urbana, inventário, semiárido

Abstract: The arborization in the central urban area of cities is a practice that beautifies urban areas and reduces anthropogenic effects in these areas. The aim of the present study was to measure and assess the quality of trees located in the medians of the city of Patos, PB, through identifying, quantifying and characterizing the vegetation in three different medians and evaluating the risks these trees posed to the population. The analyzed vegetation included 166 individual trees belonging to 14 different species, $71.4 \%$ of which were exotic species. The most represented species were Azadirachta indica and Prosopis juliflora. Most of the tress were fully grown and measured over $10 \mathrm{~cm} \mathrm{DBH}$ with heights ranging from 1.6 to $7.21 \mathrm{~m}$. In relation to risk assessment, there were no specimens that interfered with the electrical networks, but the presence of hollow branches was found in low to medium levels. In most individuals the root systems were located underground, but it was observed that $70.1 \%$ of the evaluated trees had surface roots (neilóides), increasing their susceptibility to falling. This study concluded that most of the trees in the medians of Patos city are exotic and most of these trees have suffered inappropriate silvicultural intervention.

Keywords: streets arborization, urban arboriculture, survey, semiarid

\footnotetext{
*Autor para correspondência

Recebido para publicação em 16/09/2015; aprovado em 15/10/2015

${ }^{1}$ Engenheira Florestal. Universidade Federal de Campina Grande, Campus de Patos, Paraíba. E-mail: ju.lucena18@hotmail.com

${ }^{2 ; 4}$ Professores do Programa de Pós-Graduação em Ciências Florestais, Universidade Federal de Campina Grande-UFCG, Campus de Patos, Paraíba. Av. Universitária, sn, Bairro Santa Cecília, Patos-PB. Brasil. E-mail: pcarneirosouto@yahoo.com.br; jacob_souto@uol.com.br.

${ }^{3}$ Engenheiro Florestal. Mestrando Programa de Pós-Graduação em Ciências Florestais, Universidade Federal de Campina Grande-UFCG, Campus de Patos,

Paraíba. Integrante grupo SECC-Jardim Botânico de Medellín. Autor de contato: E-mail: jdzeaca@gmail.com;

${ }^{5}$ Professor da Unidade Acadêmica de Ciências Agrárias/CCTA/UFCG, Pombal-PB. E-mail: lautersouto@yahoo.com.br
} 


\section{INTRODUÇÃO}

A arboricultura como área de trabalho no Brasil é um tema relativamente recente e de evolução lenta, que tem se desenvolvido principalmente nos últimos 30 anos após o primeiro Encontro Nacional de Arborização Urbana realizado em Porto Alegre-RS, e precisa do compromisso tanto das administrações locais como da participação dos cidadãos (MELO e ROMANINI, 2008; ROMANI et al., 2012).

Em qualquer cidade, a arborização deve ser o resultado de um planejamento criterioso, visando priorizar a flora nativa e levando em consideração todos os elementos bióticos e abióticos que fazem parte integral do ambiente urbano para garantir que não haverá conflitos futuros entre o desenvolvimento das árvores e a infraestrutura construída (MORAES e MACHADO, 2014).

Observa-se com preocupação que, mesmo com os avanços técnicos e com a facilidade de qualificação de pessoal, continua-se cometendo erros no planejamento, na implantação e manejo da arborização das cidades. As causas são, principalmente, a improvisação e aprimoramento das administrações municipais, a ausência de pessoal devidamente capacitado, o desconhecimento dos moradores que plantam sem critério técnico e a falta de trabalhos acadêmicos em arboricultura urbana (PEREIRA et al., 2011; ALENCAR et al., 2014).

Estudos sobre árvores em espaços urbanos são ferramentas úteis para os órgãos públicos tomarem decisões em relação a melhoria das áreas verdes, com a finalidade de oferecer um ambiente saudável aos cidadãos, e, a ausência ou a falta de manejo nessas áreas pode ocasionar desordem no meio urbano, com consequências danosas para o citadino (NÓBREGA et al., 2014; MARTINI et al., 2014).

A arborização viária é um elemento integrante da cobertura vegetal e sempre está presente nas cidades. Sua principal característica é que ela se desenvolve em meio da infraestrutura e das vias de trafego veicular, estando em contato permanente com os transeuntes. Dada sua localização, as árvores oferecem inúmeros benefícios ambientais, como também serviços ecossistêmicos para os cidadãos que habitem e frequentem aquelas áreas (TONETTI e NUCCI, 2012).

A composição dos canteiros centrais é diversificada, com diferenças visíveis entre grandes centros, bairros, de acordo com fatores sociais, econômicos e culturais. Na introdução de árvores nos canteiros centrais, deve-se considerar os elementos de estrutura como redes de água, esgoto, energia, e associar as exigências das plantas com as condições do local. Através do ótimo planejamento e medidas de conservação, os canteiros centrais exercem a função de corredores ecológicos, propiciando aumento na qualidade de vida para a população (DANTAS e SOUZA, 2004; BOBROWSKI et al., 2009).

Na cidade de Patos-PB, os canteiros centrais dispõem de espécies nativas e exóticas, com várias árvores em conflito com o meio urbano devido à falta de planejamento. Daí surge à necessidade de realizar estudos sobre arborização nos canteiros centrais, o que permitirá o diagnóstico que irá subsidiar ações futuras de manejo e planejamento.
O objetivo do trabalho foi quantificar e avaliar a qualidade das árvores localizadas em canteiros centrais da cidade de Patos-PB e o risco que elas representam para a população.

\section{MATERIAL E MÉTODOS}

O estudo foi realizado no ano de 2013 na cidade de Patos-PB, localizada na mesorregião sertão paraibano $\left(07^{\circ}\right.$ $01^{\text {ee }} 32^{\prime}$ S e $37^{\circ} 16^{\text {ee }} 40^{\prime}$ ), sendo o clima, segundo Köppen (1996), caracterizado como BSh quente e seco com poucas chuvas, e o bioma local é a Caatinga. A zona urbana apresenta $5,11 \mathrm{~km}^{2}$ de extensão territorial, com uma população de aproximadamente 100.674 habitantes, segundo dados do IBGE (2010).

Para o levantamento das espécies nos canteiros centrais, foram percorridos 1.391,95 $\mathrm{m}$ entre as ruas Av. Solon de Lucena, Epitácio Pessoa e Horácio Nóbrega, e para isso utilizou-se os seguintes materiais: pranchetas, fita métrica, planilhas, câmera digital, mira graduada. A identificação dos indivíduos foi realizada no momento da coleta de dados. A classificação das famílias botânicas seguiu as orientações do sistema Angiosperm Phylogeny Group (APGIII, 2009).

O levantamento qualitativo baseou-se em Silva Filho (2003), Klechowicz (2006) e Pereira et al. (2011). Os dados coletados foram: data da coleta; nome da rua, origem da espécie (consulta na literatura); nome popular e cientifico da espécie e condições do meio. As espécies foram classificadas em nativas ou exóticas baseando na literatura (Lorenzi, 2008) e sites especializados (JARDIM BOTÂNICO DO RIO DE JANEIRO, 2015).

Para os dados dendrológicos utilizou-se a classificação proposta pela COELBA (2002), sendo considerados os seguintes parâmetros: porte da árvore (pequeno: $\leq 5 \mathrm{~m}$; médio: 5-10 m; grande: > $10 \mathrm{~m}$ ), diâmetro do tronco, condições do sistema radicular, presença de pragas e doenças. Já a determinação da altura da bifurcação baseou-se em Klechowicz (2001), Silva Filho (2003) e Pereira et al. (2011), onde se utilizou a régua graduada para medir a altura da $1^{\mathrm{a}}$ bifurcação.

A avaliação de risco de queda foi feita com ênfase em localização dos canteiros levando em consideração os danos às pessoas, a presença ou ausência do neilóide e a presença de galhos ocos.

Os dados quantitativos e qualitativos foram sistematizados e processados no software Microsoft Office Excel ® 2007.

\section{RESULTADOS E DISCUSSÃO}

No levantamento quantitativo foram registrados 166 indivíduos, sendo 157 lenhosos e nove monocotiledôneas, distribuídos em 14 espécies e seis famílias botânicas (Tabela 1). As famílias que se destacaram com maior número de indivíduos foram a Meliaceae e Fabaceae, que contribuíram no total de indivíduos com $42,17 \%$ e $33,13 \%$, respectivamente. 
Tabela 1. Espécies arbóreas e respectivas famílias encontradas em canteiros centrais da cidade de Patos-PB

\begin{tabular}{|c|c|c|c|c|}
\hline Nome Vulgar & Nome científico & Família & Origem & $\mathrm{N}^{\mathrm{o}}$ Ind. \\
\hline Nim Indiano & Azadirachta indica (A. Juss) & Meliaceae & Exótica & 70 \\
\hline Algaroba & Prosopis juliflora (Sw.) DC. & Fabaceae & Exótica & 47 \\
\hline Ipê-rosa & Handroanthus impetiginosus (Mart. ex DC.) Mattos & Bignoniaceae & Nativa & 19 \\
\hline Aroeira da praia & Schinus terebinthifolius Raddi & Fabaceae & Exótica & 6 \\
\hline Craibeira & $\begin{array}{l}\text { Tabebuia aurea (Silva Manso) Benth. \& Hook. f. ex S. } \\
\text { Moore }\end{array}$ & Bignoniaceae & Nativa & 5 \\
\hline Palmeira Cica & Cycas revoluta Thunb. & Cycadaceae & Exótica & 5 \\
\hline Palmeira imperial & Roystonea oleracea (Jacq.) O.F. Cook & Arecaceae & Exótica & 4 \\
\hline Flamboyant & Delonix regia (Bojer ex Hook.) Raf. & Fabaceae & Exótica & 3 \\
\hline Cassia & Senna siamea (Lam.) H.S. Irwin \& Barneby & Fabaceae & Exótica & 2 \\
\hline Mangueira & Mangifera indica $\mathrm{L}$. & Anacardiaceae & Exótica & 1 \\
\hline Tamarindo & Tamarindus indica $\mathrm{L}$. & Fabaceae & Exótica & 1 \\
\hline Esponjinha & Albizia lebbeck (L.) Benth. & Fabaceae & Exótica & 1 \\
\hline Canafístula & Peltophorum dubium (Spreng.) Taub. & Fabaceae & Nativa & 1 \\
\hline Cajarana & Spondias lutea L. & Meliaceae & Nativa & 1 \\
\hline TOTAL & & & & 166 \\
\hline
\end{tabular}

Fonte: Lucena (2013)

Em relação a origem fitogeográfica, constatou-se que $71,4 \%$ das espécies presentes nos canteiros centrais analisados são exóticas, o que representa $84,3 \%$ dos indivíduos amostrados. Resultado ainda menos favorável foi encontrado por Bobrowski et al. (2009) em levantamento exploratório realizado sobre a composição florística em canteiros de quatro segmentos de rua da cidade de Curitiba (PR) onde foram registradas apenas quatro espécies de árvores, todas elas exóticas, o que evidencia a tendência geral das cidades brasileiras a ter dominância de espécies alienígenas em suas ruas principais.

Quanto à frequência relativa, observa-se na tabela 2, que a espécie mais ocorrente nos canteiros centrais é Azadirachta indica com 42,42\%, seguida de Prosopis juliflora (28,48\%) e Handroanthus impetiginosus $(10,90 \%)$ as demais espécies registraram frequência abaixo de $4 \%$. A espécie Azadirachta indica vem sendo muito utilizada na arborização das cidades no semiárido paraibano, devido a sua adaptabilidade e a seu rápido crescimento que proporciona sombreamento aos pedestres e veículos. Situação similar aconteceu também com a espécie Prosopis juliflora, confirmando a existência de modismo na arborização urbana onde uma determinada espécie é difundida em uma região, por um determinado período, até que se encontre outra que poderá ser bem aceita pela população.

Tabela 2. Frequência absoluta e relativa dos indivíduos presente na arborização de canteiros centrais na cidade de Patos-PB

\begin{tabular}{|c|c|c|c|}
\hline Nome Científico & Nome popular & FA & FR \\
\hline Azadirachta indica (A. Juss) & Nim & 70 & $42,42 \%$ \\
\hline Prosopis juliflora (Sw) DC & Algaroba & 47 & $28,48 \%$ \\
\hline Handroanthus impetiginosus (Mart. ex DC.) & Ipê-rosa & 19 & $10,90 \%$ \\
\hline Schinus terebinthifolius Raddi & Aroeira da praia & 6 & $3,63 \%$ \\
\hline Tabebuia aurea (Silva Manso) Benth. \& ook.f. ex S.Moore & Craibeira & 5 & $3,0 \%$ \\
\hline Cycas revoluta Thunb. & Palmeira cica & 5 & $3,0 \%$ \\
\hline Roystonea oleracea (Jacq.) O.F. Cook & Palmeira-Imperial & 4 & $2,42 \%$ \\
\hline Delonix regia (Bojer ex Hook.) Raf. & Flamboyant & 3 & $1,81 \%$ \\
\hline Senna siamea (Lam.) H.S. Irwin \& Barneby & Cassia & 2 & $1,21 \%$ \\
\hline Mangifera indica $\mathrm{L}$. & Mangueira & 1 & $0,60 \%$ \\
\hline Tamarindus indica $\mathrm{L}$. & Tamarindo & 1 & $0,60 \%$ \\
\hline Albizia lebbeck (L.) Benth. & Esponjinha & 1 & $0,60 \%$ \\
\hline Peltophorum dubium (Spreng.) Taub. & Canafístula & 1 & $0,60 \%$ \\
\hline Spondias lutea L. & Cajarana & 1 & $0,60 \%$ \\
\hline
\end{tabular}
FA: Frequência absoluta; FR: Frequência relativa.

Situação semelhante foi observada por Calixto Júnior et al. (2009) na cidade de Lavras da Mangabeira (CE), e por Alencar et al. (2014) em trabalho realizado na cidade de São João do Rio do Peixe-PB, em que a espécie Azadirachta indica foi a mais frequente, representando $23,63 \%$ e $52,59 \%$, respetivamente, do total de indivíduos arbóreos inventariados, comprovando sua prevalência na arborização de algumas cidades da região semiárida no Nordeste brasileiro.

A utilização de vários indivíduos de uma mesma espécie na arborização pode estar mais exposta ao ataque de pragas e doenças, provocando sérios danos na cobertura vegetal, minimizando assim, os benefícios proporcionados pelas árvores. Isto é referenciado por alguns autores como Melo et al. (2007) e Rodolfo Júnior et al. (2008) que além disso, aceitam a proposta de Grey e Deneke (1986) de ter no máximo 10 a $15 \%$ do total de indivíduos da mesma espécie em qualquer cidade.

No caso do Handroanthus impetiginosus, seu estabelecimento é relativamente recente, e faz parte das atividades de melhoria e adequação da arborização viária da cidade. Kulchetscki et al. (2006) afirmam que o uso de espécies nativas na arborização dos canteiros está se tornando 
mais frequente, pois apresentam fácil adaptabilidade ao meio e mantém a biodiversidade, além de criar belezas singulares e atrativas. Diefenbach e Viero (2010) relatam que este fato está se tornando uma tendência nacional, uma vez que realizado o planejamento, os benefícios serão compensatórios e não causarão implicações na estrutura das cidades futuramente.
Em relação aos dados dendrológicos (Figura 1), verificou-se que a menor média do diâmetro a altura do peito (DAP) foi registrado na espécie Handroanthus impetiginosus $(3,89 \mathrm{~cm})$, por se tratar de mudas recém-implantadas. As demais espécies apresentaram DAP acima de $10 \mathrm{~cm}$, com destaque para a espécie Prosopis juliflora com alguns indivíduos registrando $45 \mathrm{~cm}$ de DAP, indicando que se trata de vegetação adulta implantada há mais tempo.

Figura 1. Média do DAP $(\mathrm{cm})$ das espécies arbóreas presentes nos canteiros centrais da cidade de Patos-PB

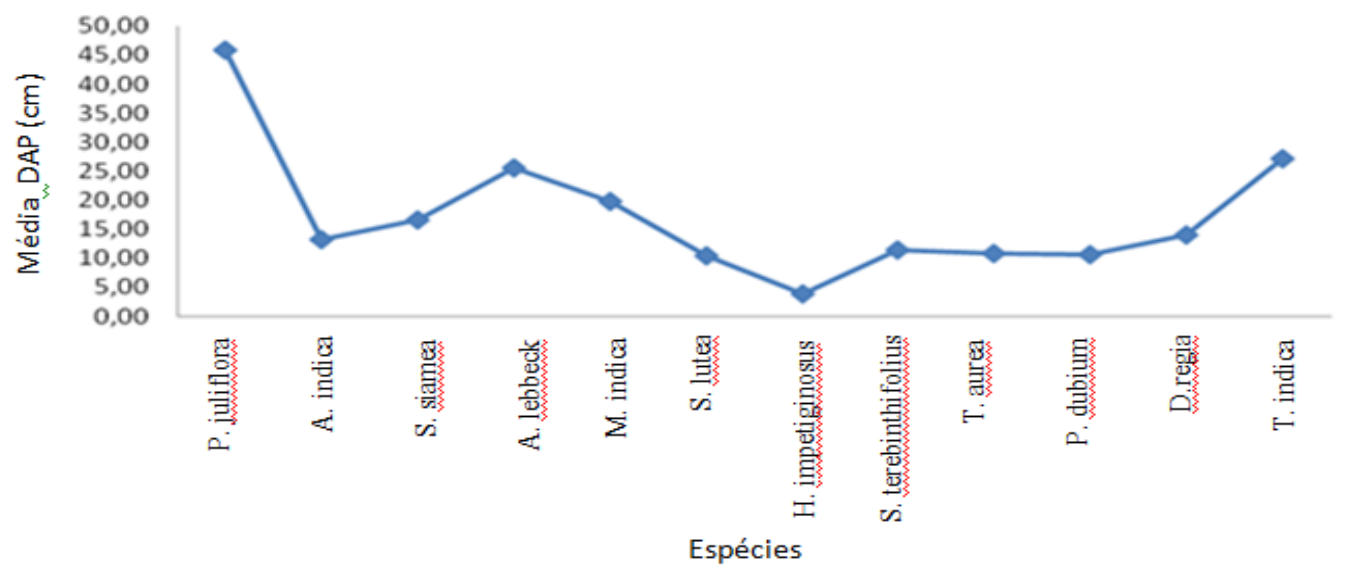

Resultado semelhante foi observado por Rodolfo Júnior et al. (2008) na cidade de Pombal-PB, onde a maioria das árvores inventariadas apresentavam DAP acima de 10,0 cm. Essa situação é comum no centro das cidades por serem os locais mais antigos, porém ressalta-se que, sempre será necessária a substituição das árvores por morte ou danos provenientes de projetos de infraestrutura, em um processo constante de renovação.

Verifica-se na figura 2 que a altura média dos indivíduos variou de 1,6 m a 7,21 m com destaque para a espécie
Prosopis juliflora que registrou o maior número de indivíduos com altura superior a 7,0 m. Segundo a classificação proposta pela COELBA (2002), grande parte dos indivíduos do presente estudo se enquadra na categoria de pequeno porte (até 5,0 m) e mediante essa característica é possível afirmar que a maioria dos indivíduos presentes na arborização dos canteiros na cidade de Patos (PB) é jovem. Além disso, segundo Melo et al. (2007), o clima da região não é favorável para o crescimento das árvores, retardando assim o seu desenvolvimento

Figura 2. Altura média (m) das espécies arbóreas presentes nos canteiros centrais da cidade de Patos-PB

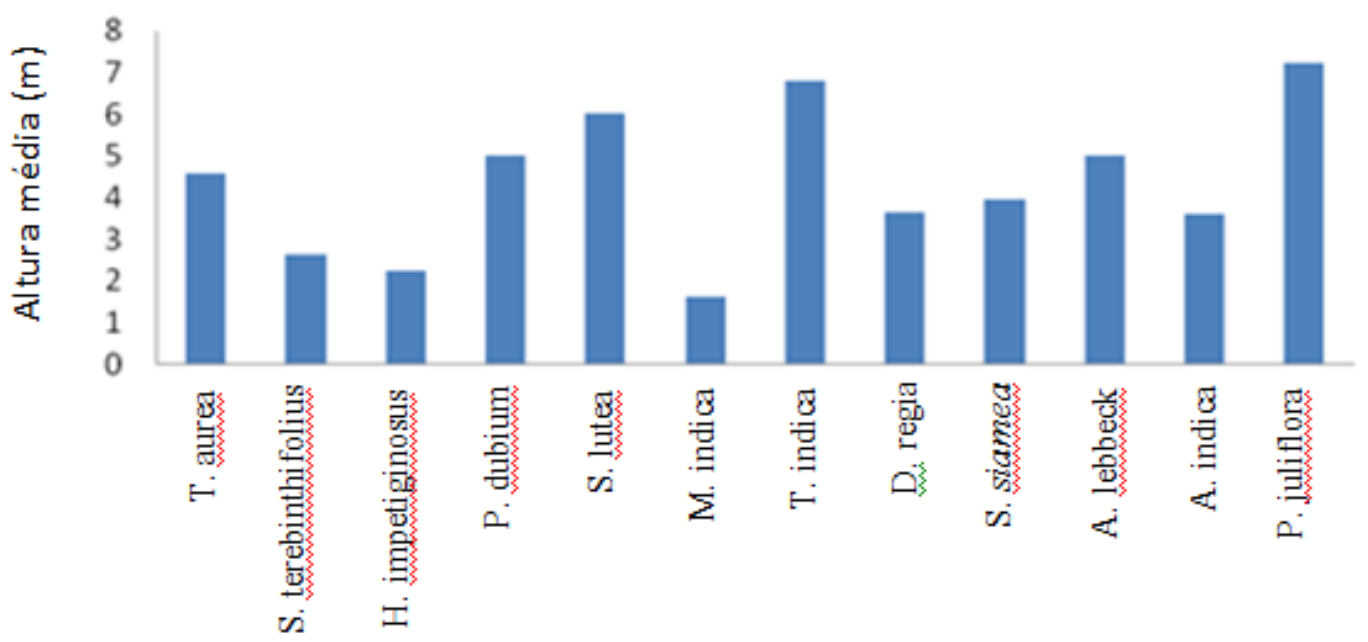

Espécies

Quanto à altura da primeira bifurcação das espécies arbóreas (Tabela 3), 95,5\% dos indivíduos mensurados apresentaram valor acima de $1,0 \mathrm{~m}$, com exceção das espécies Schinus terebinthifolius e Mangifera indica que obtiveram zero por se tratar de mudas recém-implantadas. Resultados semelhantes foram encontrados por Loboda et al. (2005) na avaliação de áreas verdes no município de Guarapuava-PR e por Barreto et al. (2014) na cidade de Valente-BA, que 
constataram a altura média da primeira bifurcação correspondente a 1,69 m e 1,55 m, respectivamente. Segundo Melo et al. (2007), a recomendação adequada dessa altura é de 2,0 m, possibilitando assim, o tráfego de pedestres. Portanto, nos casos das árvores jovens, as podas de condução devem ser realizadas, o que possibilitará seguir essa orientação técnica e, consequentemente, reduzir o uso de podas drásticas que afeta a longevidade do vegetal e é uma prática comum na maioria das cidades da região nordeste do Brasil.

Tabela 3. Frequência relativa para altura da primeira bifurcação em indivíduos presentes na arborização de canteiros centrais na cidade de Patos-PB

\begin{tabular}{lccc}
\hline \multicolumn{1}{c}{ Espécie } & Altura da primeira bifurcação (m) & Número de Indivíduos & Frequência Relativa (\%) \\
\hline Tabebuia aurea & $>1$ & 5 & 3,20 \\
Schinus terebinthifolius & 0 & 6 & 3,80 \\
Handroanthus impetiginosus & $>1$ & 19 & 12,10 \\
Peltophorum dubium & $>1$ & 1 & 0,64 \\
Spondias mombin & $>1$ & 1 & 0,64 \\
Mangifera indica & 0 & 1 & 0,64 \\
Tamarindus indica & $>1$ & 3 & 0,64 \\
Delonix regia & $>1$ & 2 & 1,90 \\
Senna siamea & $>1$ & 1 & 1,30 \\
Albizia lebbeck & $>1$ & 70 & 0,64 \\
Azadirachta indica & $>1$ & 47 & 44,60 \\
Prosopis juliflora & $>1$ & 157 & 29,90 \\
TOTAL & & 100,00 \\
\hline
\end{tabular}

Na tabela 4 são apresentados alguns parâmetros para avaliar o risco de queda dos indivíduos arbóreos. No presente estudo, constatou-se que foram realizadas podas com função meramente estética e que os indivíduos presentes nos canteiros centrais não apresentam conflito com a rede elétrica, sendo isso decorrente do uso adequado de elementos de iluminação ao longo dos canteiros. Segundo a CEMIG
(2011), é essencial essa relação para minimizar os riscos de acidentes com pedestres e eventuais interrupções no fornecimento de energia. De acordo com Schallenberger e Machado (2013), a poda eficiente das árvores é a melhor maneira de garantir o aspecto vigoroso das mesmas, favorecendo seu crescimento e evitando conflitos com os elementos de infraestrutura das cidades.

Tabela 4. Parâmetros para avaliação do risco de queda das espécies arbóreas encontradas em canteiros centrais da cidade de Patos-PB

\begin{tabular}{|c|c|c|c|c|}
\hline Espécie & $\begin{array}{c}\text { Frequência } \\
\text { Relativa }(\%)\end{array}$ & Neilóides & Galhos ocos & Podas \\
\hline Tabebuia aurea & 3,20 & Ausente & Leve & Condução \\
\hline Schinus terebinthifolius & 3,80 & Ausente & Ausente & Condução \\
\hline Handroanthus impetiginosus & 12,10 & Ausente & Ausente & Não \\
\hline Peltophorum dubium & 0,64 & Ausente & Ausente & Não \\
\hline Spondias lutea & 0,64 & Ausente & Ausente & Não \\
\hline Mangifera indica & 0,64 & Ausente & Ausente & Não \\
\hline Tamarindus indica & 0,64 & Ausente & Leve & Condução \\
\hline Delonix regia & 1,90 & Ausente & Médio & Condução \\
\hline Senna siamea & 1,30 & Ausente & Ausente & Não \\
\hline Albizia lebbeck & 0,64 & Ausente & Leve & Condução \\
\hline Prosopis juliflora & 29,90 & Parcial & Médio & Condução \\
\hline Azadirachta indica & 44,60 & Ausente & Leve & Condução \\
\hline
\end{tabular}

Em relação a presença ou não de neilóides que são estruturas que auxiliam na melhor sustentação das plantas (Tabela 4), observou-se que em $70,1 \%$ dos indivíduos, o neilóide encontra-se ausente, apenas os indivíduos da espécie Prosopis juliflora apresentam esta estrutura parcialmente porque parte dela foi enterrada. Segundo Sampaio et al. (2010), essa condição evidencia o plantio em profundidade superior a preconizada nas orientações técnicas prejudicando, assim, as trocas gasosas e a expansão do sistema radicular. Isso, ao longo do tempo e, com a infiltração da água, pode provocar o apodrecimento dos neilóides e, consequentemente, a queda da árvore.

Ainda na tabela 4, em relação à presença de galhos ocos, as espécies Delonix regia e Prosopis juliflora que apresentam indivíduos adultos, receberam a classificação médio em virtude da presença de galhos ocos em maior número $(31,9 \%)$, indicando que já sofreram algum tipo ação antrópica. Já 49,0\% dos indivíduos obtiveram a classificação leve e correspondem a mudas em desenvolvimento das espécies Tabebuia aurea, Tamarindus indica, Albizia lebbeck e Azadirachta indica. Os 19,1\% dos indivíduos restantes não 
apresentam este tipo de risco. Para Pereira et al. (2011), a queda de galhos é um problema muito grave na arborização de várias cidades no mundo e deve ser atendido de forma preventiva com práticas eficazes que diminuam o risco que representa para o pedestre e para a infraestrutura, salientando a necessidade de um plano diretor e integral para plantio e manutenção das espécies arbóreas de acordo com as necessidades e particularidades de cada região ou cidade.

\section{CONCLUSÕES}

A maior parte das espécies e indivíduos encontrados nos canteiros centrais da cidade de Patos (PB) são espécies exóticas, o que suscita a necessidade de um programa de arborização mais estruturado no centro da cidade, que tenha como ponto de partida a produção e uso de espécies nativas.

Os indivíduos arbóreos apresentam boas condições fitossanitárias sendo necessária maior atenção para a espécie Prosopis juliflora que apresentou maior número de galhos ocos, em virtude de podas equivocadas, o que pode facilitar a ação de insetos e proliferação de patógenos.

A ausência de neilóides em praticamente todos os indivíduos arbóreos analisados reflete o desconhecimento na implantação das mudas, onde o coleto é totalmente enterrado, proporcionando a ausência dessas estruturas no indivíduo adulto.

Grande parte dos indivíduos localizados nos canteiros centrais sofre intervenção silvicultural de forma inadequada, o que representa risco para a população, indicando que a arborização da cidade e sua manutenção não estão sendo feitas segundo as diretrizes técnicas.

\section{REFERÊNCIAS BIBLIOGRÁFICAS}

ALENCAR, L.S; SOUTO P.C.; MOREIRA, F.T.A.; SOUTO, J.S.; BORGES, C.H.A. Inventário quali-quantitativo da arborização urbana em São João do Rio do Peixe - PB. ACSA, v.10, n.2, p.117-124, 2014.

APG III. Anup date of the Angiosperm Phylogeny Group classification for the orders and families of flowering plants: APG III. Botanical Journal of the Linnean Society, v.161, p.105-121, 2009.

BARRETO, A.M.R.; PAULA, A.; BARRETO, P.A.B.; BARRETO, M.G.M.R. Diagnóstico da arborização urbana do bairro Dionísio Mota, município de Valente, Estado da Bahia. Enciclopédia Biosfera, v.10, n.19, p.2108-2119, 2014.

BOBROWSKI, R.; BIONDI; BAGGENSTOSS. Composição de canteiros na arborização de ruas de Curitiba (PR). REVSBAU, v.1, n.1, p.44-61, 2009.

CALIXTO JÙNIOR, J.T; SANTANA, G.M; LIRA FILHO, J.A. Análise quantitativa da arborização urbana de Lavras Da Mangabeira, CE, Nordeste do Brasil). REVSBAU, v.4, n.3, p.99-109, 2009.

CEMIG. Companhia Energética de Minas Gerais. Manual de arborização. Belo Horizonte: CEMIG / Fundação Biodiversitas, 2011. 112 p.
COELBA - Companhia de Eletricidade do Estado da Bahia. Guia de Arborização Urbana. Salvador: Venturie Gráfica e Editora, 2002. 55 p.

DANTAS, I.C.; SOUZA, C.M.C. Arborização urbana na cidade de Campina Grande-PB: Inventário e suas espécies. Revista de Biologia e Ciências da Terra, v.4, n.2, 2004.

DIEFENBACH, S.S; VIERO, V.C. Cidades sustentáveis: a importância da arborização urbana através do uso de espécies nativas. In: CONGRESSO INTERNACIONAL DE SUSTENTABILIDADE E HABITAÇÃO DE INTERESSE SOCIAL, 2010. Anais... Porto Alegre-RS, 2010 .

GREY, G. W., DENEKE, F. J. Urban forestry. New York:John Wiley \& Sons, 1986. 279 p.

IBGE-Instituto de Biogeografia e Estatística de Brasil. Censo demográfico 2010.2 Disponível:em: <http://www.censo2010.ibge.gov.br/sinopse/index.php?u $\mathrm{f}=25 \&$ dados $=0>$. Acesso em: 02 jul. 2015.

JARDIM BOTÂNICO DO RIO DE JANEIRO. Flora do Brasil. 2015. Disponível em: <http://www.floradobrasil.jbrj.gov.br> Acesso em: 02 set. 2015.

KLECHOWICZ, N.A. Diagnóstico dos acidentes com árvores na cidade de Curitiba-PR. 2001. 96 f. Dissertação (Mestrado em Engenharia Florestal). Universidade Federal do Paraná, Curitiba-PR, 2001.

KULCHETSCKI, L.; CARVALHO, P.E.; KULCHETSCKI, S.S.; RIBAS, L.L.F.; GARDINGO, J.R. Arborização urbana com essências nativas: uma proposta para a região centro-sul brasileira. Revista Ciências Exatas Terra, v.12, n.03, 2006.

KÖPPEN, W. Tradução: CORRÊA, A.C.B. Sistema Geográfico dos Climas. 1996. 494 Notas e Comunicado de Geografia - Série B: Textos Didáticos nº13. Ed. 495 Universitária - UFPE, Departamento de Ciências Geográficas, UFPE, p.31.

LOBODA, C.R.; ANGELIS, B.L.D.; N.G.A.; SILVA, E.S. Avaliação das áreas verdes em espaços públicos no município de Guarapuava/PR. Revista Ambiência, v.1, n.1, p.141-155, 2005.

LORENZI, H. Árvores brasileiras: manual de identificação e cultivo de plantas arbóreas nativas do Brasil. 5.ed. v.1, Rio de Janeiro, Nova Odessa: Instituto Plantarum, 368 p. 2008.

MARTINI, A.; GASPAR, R.G.B.; BIONDI, D. Diagnóstico da implantação da arborização de ruas no bairro Santa Quitéria, Curitiba - PR. REVSBAU, v.9, n.2, p 148-167, 2014. 
MELO, E.F.R.Q.; ROMANINI, A. Praça Ernesto Tochetto: importância da sua preservação histórica e aspectos de sua arborização. REVSBAU, v.3, n.1, p. 54-72, 2008.

MELO, R.R.; LIRA FILHO, J.A.; RODOLFO JÚNIOR, F. Diagnóstico qualitativo e quantitativo da arborização urbana no bairro Bivar Olinto, Patos, Paraíba. REVSBAU, v.2, n.1, p. 64-80, 2007.

MINHOTO, E.S; MONTEIRO, E.A; FISCH, S.T.V. Arborização viária na cidade de Taubaté, SP: no centro comercial histórico e um bairro residencial moderno. REVSBAU, v.4, n.2, p.82-96, 2009.

MORAES, L.A., MACHADO, R.R.B. A arborização urbana do município de Timon/MA: inventário, diversidade e diagnóstico quali-quantitativo. REVSBAU, v.9, n.4, p 80-98, 2014.

NÓBREGA, C.C.; SOUTO, P.C.; ARAÚJO, L.H.B.; SILVA, A.C.F.; PINTO, M.G.C.. Análise quanti-qualitativa das espécies arbóreas presentes no parque religioso Cruz da Menina, Patos/PB. ENCICLOPÉDIA BIOSFERA, v.10, n.18, p. 299-307, 2014.

PEREIRA, P.H.; TOPANOTTI, L.R., DALLACORT, S., MOTA, C.J., BRUN, F.G.K., SILVA, R.T.L. Estudo de caso do risco de queda de árvores urbanas em via pública na cidade de Dois Vizinhos-PR. Synergismus scyentifica UTFPR, v.6, n.1, 2011.

RODOLFO JÚNIOR, F., MELO, R.R., CUNHA, T.A., STANGERLIN, D.M. Análise da arborização urbana em bairros da cidade de Pombal no estado da Paraíba. REVSBAU, v.3, n.4, p.3-19, 2008.

ROMANI, G.N.; GIMENES, R.; SILVA, M.T.; PIVETTA K.F.L.; BATISTA, G.S. Análise quali-quantitativa da arborização na praça XV de novembro em Ribeirão Preto - SP, Brasil. Revista Árvore, v.36, n.3, p.479-487, 2012.

SAMPAIO, A.C.F.; DUARTE, F.G.; SILVA, E.G.C.; ANGELIS, B.L.D.; BLUM, C.T. Avaliação de árvores de risco na arborização de vias públicas de Nova Olímpia, Paraná. REVSBAU, v.5, n.2, p.82-104, 2010.

SCHALLENBERGER, L.S; MACHADO, G.O. Inventário da arborização na região central do município de Mangueirinha-PR. REVSBAU, v.8, n.1, p.54-64, 2013.

SILVA FILHO, DF. Silvicultura urbana: o desenho florestal da cidade. Departamento de Ciências Florestais da ESALQ/USP, 2003. Disponível em: <http://www.ipef.br/silvicultura/urbana.asp> Acesso em: 05 set. 2013.

TONETTI, E.L.; NUCCI, J.C. Arborização viária na área central de Paranaguá (PR) Brasil. Revista Geografar, v.7, n.1, p. 53-67, 2012. 